\title{
Leclercia adecarboxylata: An Emerging Pathogen Among Pediatric Infections
}

\author{
Jonathan Keyes $^{1}$, Evan P. Johnson ${ }^{2}$, Monica Epelman ${ }^{3}$, Adriana Cadilla ${ }^{4}$, Syed Ali ${ }^{5}$
}

1. Internal Medicine, University of Central Florida College of Medicine, Orlando, USA 2. Orthopaedics, University of Central Florida College of Medicine, Orlando, USA 3. Radiology, Nemours Children's Hospital/University of Central Florida College of Medicine, Orlando, USA 4. Infectious Disease, Nemours Children's Hospital, Orlando, USA 5. Inpatient Pediatrics, Nemours Children's Hospital, Orlando, USA

Corresponding author: Syed Ali, syed.ali@nemours.org

\begin{abstract}
Leclercia adecarboxylata is a gram-negative bacillus of the Enterobacteriaceae family. It is a rare human pathogen that is often acquired via wound and/or contact with aquatic environment. Although multiple cases of $L$. adecarboxylata infections are described in the adult population, few have been documented in pediatrics. We will present two cases of $L$. adecarboxylata infections in the pediatric population. The first is a case of cellulitis in an 11-year-old male patient after a penetrating wound. The second is a first-documented urinary tract infection in a 16-year-old male patient with chronic kidney disease. Both patients were successfully treated with antibiotics and surgical intervention, if necessary. These cases highlight the growing emergence of this bacterium in the pediatric population and the need to become more aware of its threat even in patients who are immunocompetent.
\end{abstract}

Categories: Pediatrics, Infectious Disease, Public Health

Keywords: leclercia adecarboxylata, pediatrics, infections, bacteria, cellulitis, urinary tract infection, emerging, chronic kidney disease

\section{Introduction}

Leclercia adecarboxylata is a mobile gram-negative bacillus that is generally sensitive to most antibiotics and was first described by Leclerc in 1962 as Escherichia adecarboxylata, but was reclassified as $L$.

adecarboxylata after further studies showed that it belonged to a different genus [1,2]. It is an extremely rare human pathogen that commonly infects immunocompromised individuals, with few cases documented in adults and even fewer in pediatrics. Of the documented pediatric cases, L. adecarboxylata is known to cause cellulitis from wound infections and/or contact with contaminated water, consistent with multiple known cases in the adult counterpart [3-7]. However, it has also been documented in pediatrics to cause folliculitis, peritonitis from peritoneal dialysis, and bacteremia in preterm infants and a patient with acute lymphoblastic leukemia [8-12]. Nonetheless, compared to adults, there are fewer cases of $L$. adecarboxylata documented in pediatrics. We present two cases to highlight the need for growing awareness of $L$. adecarboxylata as it is becoming an emerging infection in the pediatric population. The first is a classic case of cellulitis after a penetrating foot infection in an immunocompetent boy and the second is a firstdocumented urinary tract infection (UTI) in a patient with chronic kidney disease (CKD).

Received 03/18/2020

began $03 / 22 / 2020$ Published 05/10/2020

\section{๑) Copyright 2020}

Keyes et al. This is an open access article distributed under the terms of the Creative Commons Attribution License CC-BY 4.0., which permits unrestricted use, distribution, and reproduction in any medium, provided the original author and source are credited.

\section{Case Presentation}

The first case is an 11-year-old boy with no past medical history who fell off his bike, hitting a branch, and sustained a penetrating wound by a wood splinter on the dorsal right foot. The mother cleaned the wound with water. The swelling persisted without drainage overnight and the next day, he went to the emergency department (ED) and was discharged on oral cephalexin to prevent cellulitis. After two days of antibiotic, the swelling worsened with draining yellow pus. Throughout this time period, the mother has continued to wash the wound with water and has noticed pieces of wood coming out. The patient was hospitalized after failing outpatient treatment. He was afebrile at the time of admission. Initial lateral radiograph of the right foot (Figure 1) on admission showed focal soft tissue swelling overlying the dorsum of the foot, but no radiopaque foreign body was identified at the time. The patient was empirically started on intravenous (IV) clindamycin. Pus specimen grew $L$. adecarboxylata that was resistant to ampicillin but sensitive to ampicillin/sulbactam, cefazolin, gentamicin, and tobramycin. Because the patient was experiencing optimal clinical response to IV clindamycin, it was decided by infectious disease to continue IV clindamycin with the eventual transition to oral clindamycin upon discharge. However, two weeks after discharge, the wound was draining again. 


\section{Cureus}

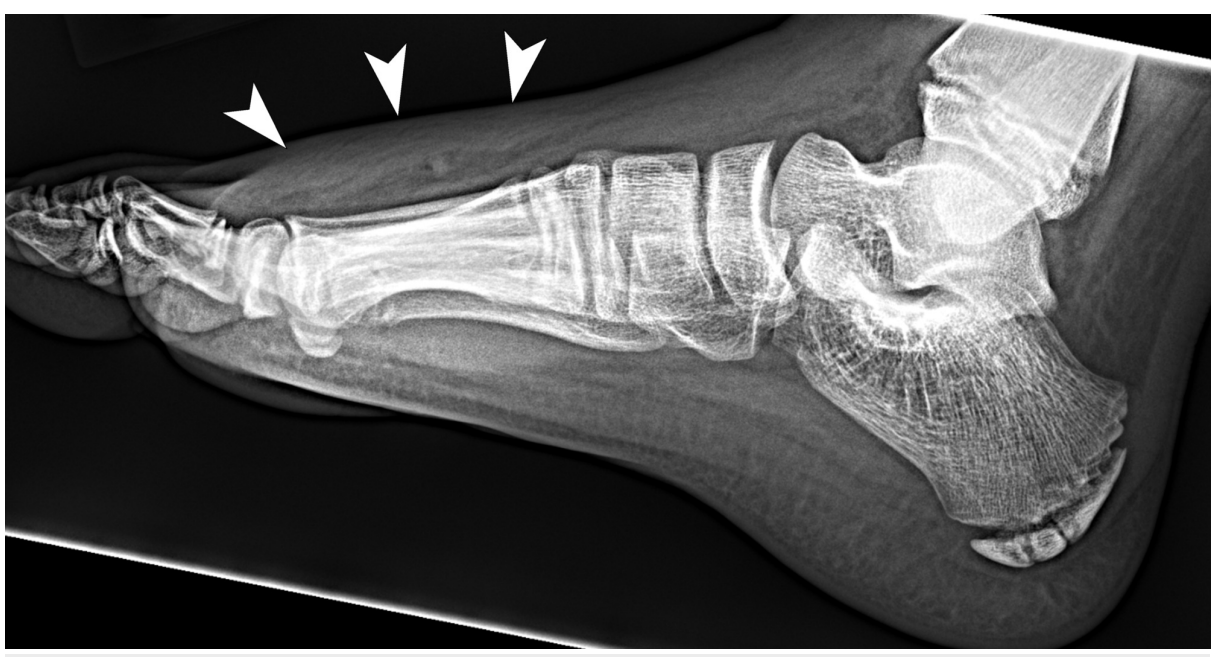

FIGURE 1: Lateral radiograph of the right foot

Initial lateral radiograph of the right foot shows focal soft tissue swelling (arrowheads) overlying the dorsum of the foot but no radiopaque foreign body.

An ultrasound (Figure 2) at the ED showed a $0.5 \times 0.8 \times 2.8 \mathrm{~cm}$ echogenic structure, suspected to be a retained foreign body or granulation tissue. Due to the persistent nature of the wound, the patient was referred to orthopedics for a wound exploration and debridement. Granulation tissue was debrided. No obvious foreign body was encountered. Intraoperative culture was negative for organisms, and the patient was discharged home with oral cephalexin. He had no further complications at follow-up.
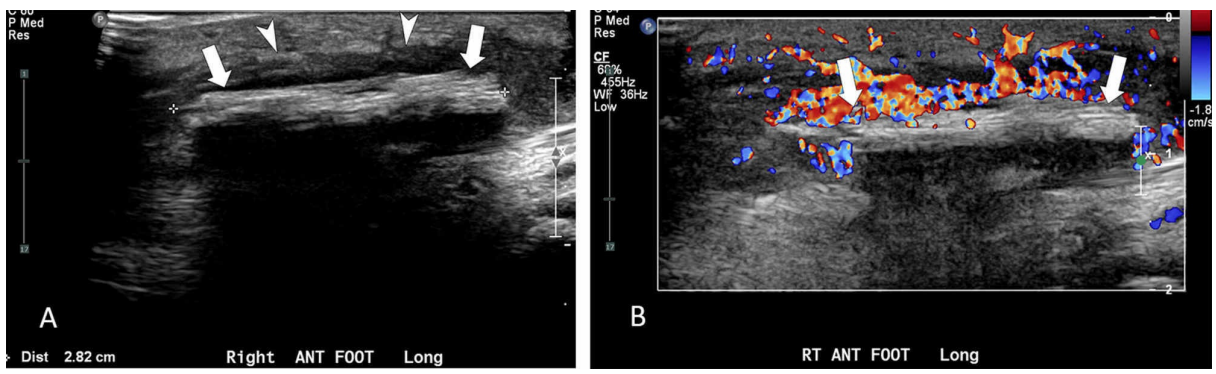

FIGURE 2: Longitudinal greyscale and color Doppler of the right foot

Longitudinal grayscale (A) and color Doppler (B) ultrasound images obtained on re-admission show an echogenic structure (arrows), believed to represent a foreign body measuring approximately $2.8 \mathrm{~cm}$ in length and located approximately $10 \mathrm{~mm}$ deep to the skin surface. Hypoechoic granulation tissue (arrowheads in A) is seen adjacent to the foreign body, which is hypervascular on color Doppler interrogation (B).

The second case is a 16-year-old male with a history of high-grade reflux nephropathy, solitary left kidney, and neurogenic bladder on intermittent Foley catheterization every three hours. The patient had stage 4 CKD with an elevated serum creatinine of $6.1 \mathrm{mg} / \mathrm{dL}$ (baseline $3.0 \mathrm{mg} / \mathrm{dL}$ ) in December 2016, which warranted the placement of a left nephrostomy tube. The nephrostomy tube was removed in March 2017. He presented to the ED with nausea and vomiting four days after left nephrostomy tube removal. There was no trauma or contact with contaminated water. He was afebrile and denied flank pain. The nephrostomy site did not appear to be infected. Creatinine was $5.0 \mathrm{mg} / \mathrm{dL}$ and blood urea nitrogen (BUN) was $55 \mathrm{mg} / \mathrm{dL}$. The mother was concerned for pyelonephritis, but ultrasound at the time did not show any significant changes in the left kidney. A midstream urine culture grew 70,000 cfu/mL L. adecarboxylata. Susceptibility panel showed that it was sensitive to ampicillin, ampicillin/sulbactam, cefazolin, gentamicin, nitrofurantoin, and tobramycin. The patient was discharged home with oral cephalexin. A week later, the patient presented back at the ED with left flank pain and intermittent vomiting, but no fever. He was not compliant with the antibiotic regimen due to complex social situation. He was hospitalized and started on IV ceftriaxone. Creatinine improved at $4.3 \mathrm{mg} / \mathrm{dL}$, BUN remained elevated at $53 \mathrm{mg} / \mathrm{dL}$, and albumin was decreased at 3.5 $\mathrm{g} / \mathrm{dL}$. Urinalysis was significant for 2+ proteinuria, but absence of white blood cells, leukocyte esterase, nitrite, and bacteria. Ultrasound (Figure 3) showed a hypodense mass in the left mid-kidney concerning for a possible abscess or a callous reaction to prior nephrostomy. Urine culture was negative for organisms, and the patient no longer experienced any left flank pain after 24 hours. He was afebrile and denied nausea and vomiting. The patient was discharged home on cefdinir. There was no symptomatic recurrence, and renal 


\section{Cureus}

ultrasound at follow-up was stable.

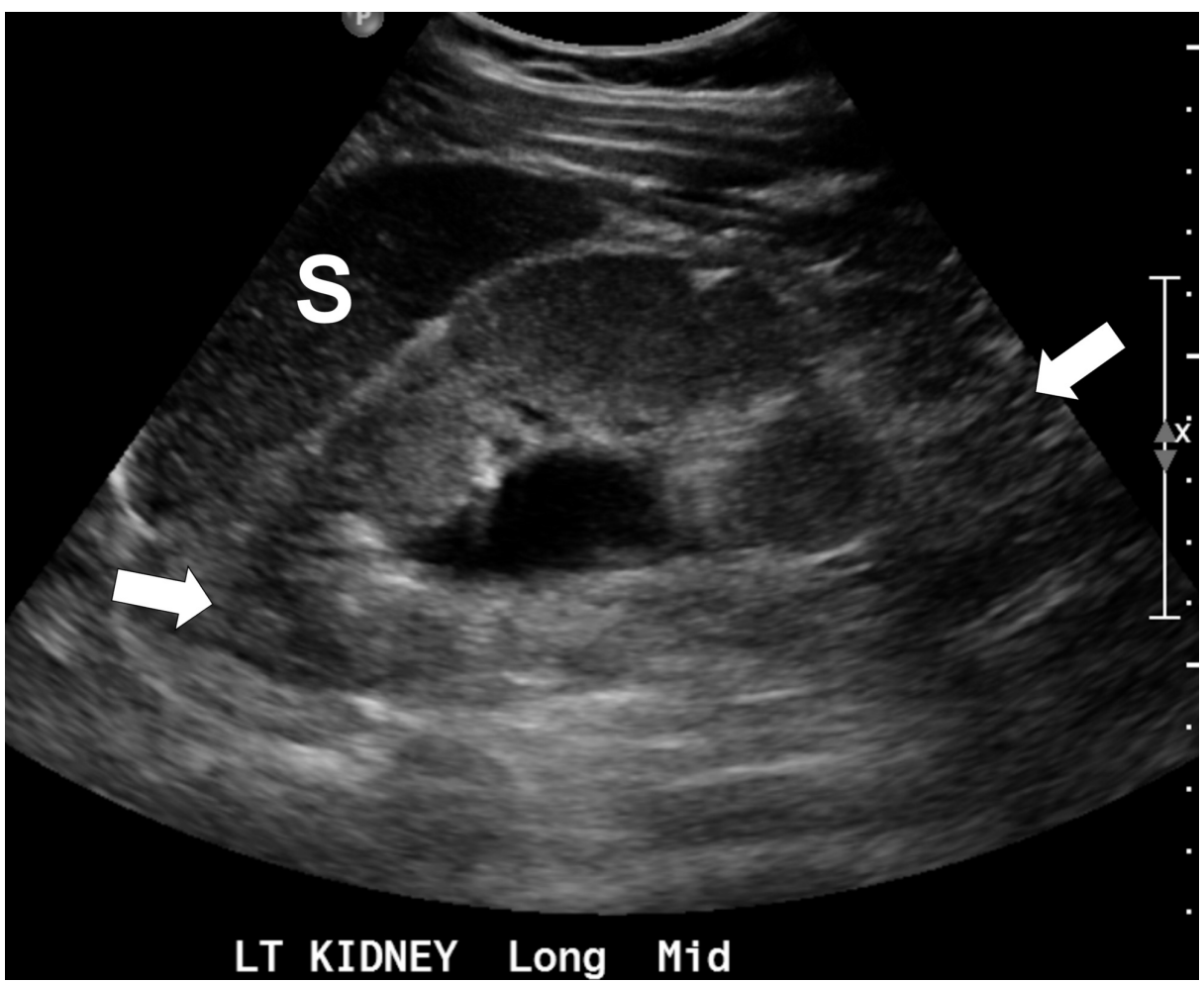

\section{FIGURE 3: Longitudinal ultrasound of the left kidney}

Longitudinal ultrasound image of the left kidney (arrows) shows a top normal size kidney with mild pelviectasis, and mild, diffuse increase in parenchymal echogenicity with poor corticomedullary differentiation. The renal parenchyma appears mildly increased in echogenicity when compared to the adjacent splenic (S) parenchyma.

\section{Discussion}

L. adecarboxylata is a human pathogen that is infrequently documented in the adult population and even less so in pediatrics. Although L. adecarboxylata was first described in 1967, it only began to generate attention in pediatric infectious disease during the past 10 years [1]. A literature review of cases published in English on PubMed using the search term "Leclercia adecarboxylata" showed 44 cases of $L$.

adecarboxylata adult infections and only nine pediatric infections. Of the documented pediatric infections, two were between 2000 and 2004, four between 2010 and 2014, and five between 2015 and 2019 (including the two cases reported here). Although L. adecarboxylata is still a relatively uncommon infection, there has been an increase in the number of reported pediatric cases throughout the recent years. Indeed, it has been speculated that the number of $L$. adecarboxylata infections could be under-reported given the high degree of phenotypic overlap between $L$. adecarboxylata and Escherichia coli and the inability for an automated diagnostic system to reliably distinguish between the two [6].

L. adecarboxylata was documented as an opportunistic pathogen that frequently causes monomicrobial infections in the immunocompromised individuals [13]. While this is true as most of the documented pediatric cases are of patients who were immunocompromised (Table 1), it is also worth noting that infections are gradually occurring in the immunocompetent patients such as our first patient presenting with cellulitis. Three other cases have been documented in pediatric patients with intact immune function: a nine-year-old girl with cellulitis after a left foot puncture wound, a two-year-old boy with cellulitis after a right thumb laceration, and a 12-year-old boy with folliculitis after exposure to aquatic environment $[3,4,8]$. Based on these case reports, the cause of infection in our patient either stemmed from retained foreign body secondary to penetrating wound or improper wound cleaning with contaminated water. The patient was exposed to both risk factors, and it is difficult to distinguish the underlying cause of his infection. Although our patient was suspected to have a retained foreign object on re-admission based on the ultrasound (Figure 2), no obvious foreign body was encountered during wound exploration. We suspect that the echogenic structure was likely to be granulation tissue and that healing had already taken place, especially since intraoperative culture did not show any organisms. Our first case is presented to raise awareness of $L$. adecarboxylata in pediatric infections and to demonstrate its emerging presence even among those who are immunocompetent. 


\section{Cureus}

\begin{tabular}{|l|l|ll|}
\hline Immune Deficiency/Underlying Condition & Culture Source & Coinfection(s) & Reference \\
\hline None & Wound & None & Grantham et al. [3] \\
None & Wound & None & Hurley et al. [4] \\
None & Folliculitis & None & Broderick et al. [8] \\
End-stage renal disease & Peritoneal fluid & None & Fattal and Deville [9] \\
Premature birth & Blood & None & Myers et al. [10] \\
Acute lymphoblastic leukemia & Blood & None & Nelson et al. [11] \\
Leukopenia and neutropenia & Blood & Staphylococcus aureus & Longhurst and West [12] \\
Acute lymphoblastic leukemia & Blood & None & Sethi et al. [14] \\
\hline
\end{tabular}

\section{TABLE 1: Features of Leclercia adecarboxylata pediatric infection case reports}

A review of all documented L. adecarboxylata infections published in English on PubMed for the pediatric population.

To our knowledge, the second case is the first published L. adecarboxylata UTI in a pediatric patient. $L$. adecarboxylata infections have been reported in the adult population after foreign body placements such as a central line or a peritoneal catheter $[16,17]$. Similarly, a child was infected with L. adecarboxylata during peritoneal dialysis for end-stage renal disease [9]. Our patient had a chronic history of intermittent Foley catheterization due to neurogenic bladder, which could have increased his risk of UTI from $L$. adecarboxylata. At the time of his first admission, we did not find evidence of pyelonephritis given the unremarkable kidney ultrasound. On re-admission, pyelonephritis was suspected based on the significant change in renal ultrasound (Figure 3). This suspicion was further supported by his uncontrolled UTI resulted from medication non-compliance and possibly inadequate immune response secondary to CKD. We know from past reports that $L$. adecarboxylata can act as an opportunistic pathogen for the immunocompromised individuals, with one case where mortality was observed in an immunocompromised pediatric patient $[13,14]$. CKD has been known for its association with acquired immunosuppression. While the patient did not demonstrate neutropenia, his state of uremia can still contribute to an inadequate immune response given that uremia can inhibit immune cell activation [18]. The patient also demonstrated proteinuria on the second admission. Unfortunately, his immunoglobulin level was not trended between the two admissions. Nonetheless, we have a high clinical suspicion that the patient may have lost immunoglobulin through urinary excretion in the past. This may have also increased his risk to $L$. adecarboxylata infection. The case again highlights the need for growing awareness of $L$. adecarboxylata infections in the pediatric population. More importantly, given the increased frequency of intermittent catheterization and nephrostomy as a result of congenital kidney diseases, it is imperative to be cognizant of $L$. adecarboxylata in this more vulnerable patient population in order to obtain an early diagnosis for proper treatment and minimize mortality.

\section{Conclusions}

We present two cases of $L$. adecarboxylata infections in the pediatric population, one who was immunologically intact with cellulitis and one who was immunologically weakened with UTI. Diagnosis was made based on laboratory findings. Proper antibiotic treatments and surgical intervention, if necessary, were provided with successful resolution of the infection in both cases. The cases highlight the emerging presence of $L$. adecarboxylata among pediatric infections and care providers should increase their awareness of this phenomenon. .

\section{Additional Information \\ Disclosures}

Human subjects: Consent was obtained by all participants in this study. Conflicts of interest: In compliance with the ICMJE uniform disclosure form, all authors declare the following: Payment/services info: All authors have declared that no financial support was received from any organization for the submitted work. Financial relationships: All authors have declared that they have no financial relationships at present or within the previous three years with any organizations that might have an interest in the submitted work. Other relationships: All authors have declared that there are no other relationships or activities that could appear to have influenced the submitted work. 


\section{References}

1. Anuradha M: Leclercia adecarboxylata isolation: case reports and review . J Clin Diagn Res. 2014, 8:3-4. 10.7860/JCDR/2014/9763.5260

2. Tamura K, Sakazaki R, Kosako Y, Yoshizaki E: Leclercia adecarboxylata gen. nov., comb. nov., formerly known as Escherichia adecarboxylata. Curr Micobiol. 1986, 13:179-184.

3. Grantham WJ, Funk SS, Schoenecker JG: Leclercia adecarboxylata musculoskeletal infection in an immune competent pediatric patient: an emerging pathogen?. Case Rep Orthop. 2015, 2015:160473. $10.1155 / 2015 / 160473$

4. Hurley EH, Cohen E, Katarincic JA, Ohnmacht RK: Leclercia adecarboxylata infection in an immunocompetent child. R I Med J. 2015, 98:41-44.

5. Hess B, Burchett A, Huntington MK: Leclercia adecarboxylata in an immunocompetent patient. J Med Microbiol. 2008, 57:896-898. 10.1099/jmm.0.47673-0

6. Michael Z, McGann PT, Alao O, Stevenson L, Lesho E, Viscount H: Isolation of Leclercia adecarboxylata from an infected war wound in an immune competent patient. Mil Med. 2013, 178:390-393. 10.7205/milmed-d-12-00382

7. Temesgen Z, Toal DR, Cockerill FR 3rd: Leclercia adecarboxylata infections: case report and review . Clin Infect Dis. 1997, 25:79-81. 10.1086/514514

8. Broderick A, Lowe E, Xiao A, Ross R, Miller R: Leclercia adecarboxylata folliculitis in a healthy swimmer: an emerging aquatic pathogen?. JAAD Case Rep. 2019, 5:706-708. 10.1016/j.jdcr.2019.06.007

9. Fattal O, Deville JG: Leclercia adecarboxylata peritonitis in a child receiving chronic peritoneal dialysis . Pediatr Nephrol. 2000, 15:186-187. 10.1007/s004670000436

10. Myers KA, Jeffery RM, Lodha A: Late-onset Leclercia adecarboxylata bacteraemia in a premature infant in the NICU. Acta Paediatr. 2012, 101:37-39. 10.1111/j.1651-2227.2011.02431.x

11. Nelson MU, Maksimova Y, Schulz V, Bizzarro MJ, Gallagher PG: Late-onset Leclercia adecarboxylata sepsis in a premature neonate. J Perinatol. 2013, 33:740-742. 10.1038/jp.2013.34

12. Longhurst CA, West DC: Isolation of Leclercia adecarboxylata from an infant with acute lymphoblastic leukemia. Clin Infect Dis. 2001, 32:1659. 10.1086/320539

13. Spiegelhauer MR, Andersen PF, Frandsen TH, Nordestgaard RLM, Andersen LP: Leclercia adecarboxylata: a case report and literature review of 74 cases demonstrating its pathogenicity in immunocompromised patients. Infect Dis. 2019, 51:179-188. 10.1080/23744235.2018.1536830

14. Sethi K, Barker EM, Metlay LA, Caserta MT, Daugherty LE: Leclercia adecarboxylata sepsis and cerebral herniation. J Pediatric Infect Dis Soc. 2014, 3:1-3. 10.1093/jpids/pis131

15. Shah A, Nguyen J, Sullivan LM, Chikwava KR, Yan AC, Treat JR: Leclercia adecarboxylata cellulitis in a child with acute lymphoblastic leukemia. Pediatr Dermatol. 2011, 28:162-164. 10.1111/j.1525-1470.2011.01348.x

16. De Mauri A, Chiarinotti D, Andreoni S, Molinari GL, Conti N, De Leo M: Leclercia adecarboxylata and catheter-related bacteraemia: review of the literature and outcome with regard to catheters and patients. J Med Microbiol. 2013, 62:1620-1623. 10.1099/jmm.0.059535-0

17. Adapa S, Konala VM, Nawaz F, et al.: Peritonitis from Leclercia adecarboxylata: an emerging pathogen . Clin Case Rep. 2019, 7:829-831. 10.1002/ccr3.2094

18. Kurts C, Panzer U, Anders HJ, Rees AJ: The immune system and kidney disease: basic concepts and clinical implications. Nat Rev Immunol. 2013, 13:738-753. 10.1038/nri3523 